\title{
Power Loading and Spectral Efficiency Comparison of MIMO OFDM/FBMC for Interference Alignment Based Cognitive Radio Systems
}

\author{
Mohammed El-Absi*, Musbah Shaat**, Faouzi Bader ${ }^{+}$and Thomas Kaiser* \\ ${ }^{*}$ Institute of Digital Signal Processing, Duisburg-Essen University, Germany \\ ${ }^{* *}$ Centre Tecnològic de Telecomunicacions de Catalunya (CTTC), Spain \\ ${ }^{+}$SUPELEC, Rennes-France \\ Emails: \{mohammed.el-absi,thomas.kaiser\}@uni-due.de,mshaat@cttc.es, faouzi.bader@supelec.fr
}

\begin{abstract}
Interference alignment (IA) has been proposed to optimally manage the interference aiming at providing the maximum degrees of freedom for multiuser interference channels. Therefore, IA has been used in cognitive radio (CR) systems to perform resource management in order to improve the throughput of the OFDM/FBMC based MIMO CR systems. In this work, a sub-optimal IA based power loading method is proposed for OFDM/FBMC based MIMO CR systems to approach the optimal approach with fewer complexity. In the proposed algorithm, all secondary users are enabled to share the available spectrum on the base of IA technique without affecting the quality-ofservice of the primary system. Furthermore, spectral efficiency comparison between MIMO-OFDM and MIMO-FBMC is presented. Simulation results show that IA based power loading achieves a significant sum-rate increase of CR systems compared to traditional orthogonal multiple access techniques. Additionally, IA based power loading achieves better sum-rate improvement with FBMC than OFDM physical layer.
\end{abstract}

Keywords-Cognitive radio, Interference alignment, FBMC, OFDM, MIMO, Power loading.

\section{INTRODUCTION}

Recently, frequency spectrum scarcity has become a serious concern due to the increasing demand of the wireless data traffic. In order to overcome the problem, cognitive radio (CR) has been proposed to utilize the temporal/spatial unused frequency bands. CR allows a group of secondary users (SUs) to access the vacant bands originally licensed to the primary users (PUs). The SU access should not alter the operation of the primary system nor cause harmful to it. Multiple-input and multiple-output (MIMO) technology and multicarrier communications have been recognized as desirable candidates for CR systems, where MIMO significantly improves the spectral efficiency by sending independent data streams simultaneously over multiple antennas, while multicarrier communications offer an important flexibility in allocating different resources between users and subcarriers [1].

Although that orthogonal frequency division multiplexing (OFDM) is the most common multicarrier technique that is considered by several communication standards, including IEEE 802.22 TV based CR system, there are several factors that limit the achieved capacity in OFDM systems. OFDM has large frequency domain sidelobes that cause high mutual 978-1-4799-5863-4/14/\$31.00 (c) 2014 IEEE interference to the adjacent primary bands. Additionally, the overall spectrum efficiency of the OFDM system is reduced due to the use of the cyclic prefix (CP) that is added to combat the multipath propagation effect. Filter bank multicarrier (FBMC) overcomes the OFDM limitations and is considered as an alternative scheme to OFDM in CR networks. In FBMC, channels are designed in the frequency domain to have the required spectral containment without any need to use $\mathrm{CP}$ as in OFDM [2]-[4].

Resource allocation approaches have been proposed in CR networks to maximize the SUs throughput while satisfying the quality-of-service (QoS) of the PUs. The problem of resource allocation for CR networks with single antenna employed at both PUs and SUs has been widely considered (e.g. [5], [6] and references therein). Furthermore, there have been many studies on CR networks with multiple antennas at the SUs in order to achieve CR regulations by cognitive beamforming (e.g. [1], [7]). Recently, a cooperative paradigm for CR networks has been proposed for CR network with multiple antennas at the PUs and SUs in order to utilize the MIMO advantage to cooperatively relay the traffic for the PUs (e.g. [8]-[10]).

Interference alignment (IA) is a powerful tool that is used to manage interference in multiuser wireless communications [11]. Therefore, resource allocation on the base of IA has been recently considered in order to improve the spectral efficiency of CR networks [12]-[16]. Meanwhile, most of the existing works of IA in CR have considered MIMO employment on both of PUs and SUs in order to allow a SU to utilize the free and non-free eigenmodes of PU. In this scenario, MIMO employment at the PU provides extra degrees of freedom to null the interference at the PU receiver and to exclude interference constraints from the optimization problem (e.g. [12]-[14]).

In this work, a sub-optimal IA based power loading among the SUs is proposed in order to improve the CR system spectral efficiency with OFDM and FBMC physical layers with acceptable computational complexity. SU nodes with MIMO employment is assumed while each PU is assumed to have a single antenna, which means that the spatial degree-offreedoms are not available anymore at PU side. The fairness among the SUs is guaranteed as well as the interference and power constraints. Performance evaluation of the proposed algorithm is presented in MIMO-OFDM and MIMO-FBMC based CR systems. 


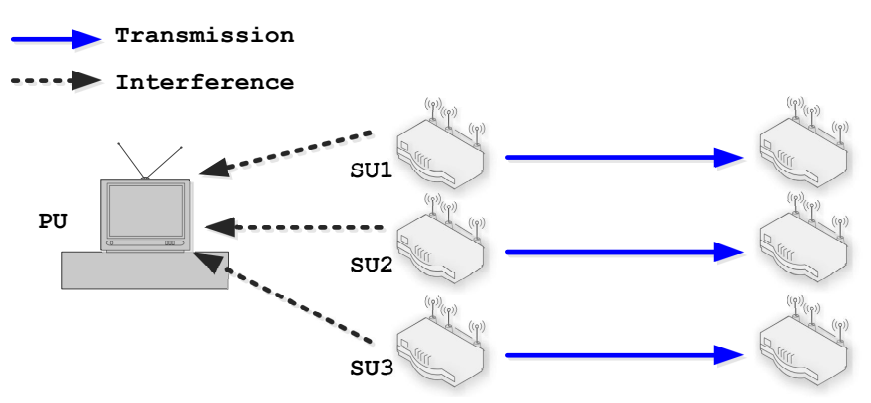

Fig. 1: Cognitive radio system.

In the following section, the system is modeled with MIMO-OFDM and MIMO-FBMC physical layers. Section III presents the optimal IA based power loading optimization problem. In Section IV, the proposed low-complexity power loading algorithms is showed. Simulation results are illustrated and discussed for multicarrier based CR systems in Section V. Finally, Section VI concludes the paper.

\section{System Model}

We consider a MIMO cognitive system with $K$ pairs of secondary transmitters and receivers with $M_{T}$ and $M_{R}$ transmit and receive antennas, respectively. This CR system operates in the same geographical area of a conventional primary network with several single antenna PUs as seen in Fig.1. The available spectrum is divided into $N$ sub-carriers with a $\triangle f$ separation, where $L$ active primary bands $\left(W_{1}, W_{2}, \ldots, W_{L}\right)$ have been occupied by the PUs. The CR system is allowed to use the active and non-active bands under a condition that the total induced interference to the $l^{\text {th }}$ PU active band is below the interference limit, $I_{t h}^{l}$.

In this work, OFDM and FBMC physical layers are considered. Therefore, a short description of them is given in this section.

\section{A. OFDM System Model}

A general block diagram of an OFDM system can be found in Fig. 2. Firstly, the bits are mapped into complex symbols. Then, the time domain samples of an OFDM symbol are generated using the inverse discrete Fourier transform (IDFT). After that, the CP is added to form the transmitted signal. Assume that $\Phi^{n}$ is the power spectrum density (PSD) of the $n^{\text {th }}$ subcarrier. In OFDM system with rectangular pulse of length $T_{s}=N+C$, where $C$ is the length of the CP, $\Phi^{n}(f)$ can be written as follows

$$
\Phi^{n}(f)=P^{n}\left(T_{s}+2 \sum_{r=1}^{T_{s}-1}\left(T_{s}-r\right) \cos (2 \pi f r)\right) .
$$

where $P^{n}$ is the power transmitted over the $n^{\text {th }}$ subcarrier.

\section{B. FBMC System Model}

In FBMC, the transmultiplexer configuration is adopted using the synthesis filter bank (SFB) at the transmitter side, and the analysis filter banks (AFB) at the receiver side as described in Fig. 2 [2], [3]. In FBMC systems, the use of critically sampled filter banks is problematic, since the aliasing effects would make it difficult to compensate imperfections of the channel by processing the sub-channel signals while the FBM$\mathrm{C}$ with the offset quadrature amplitude modulation(OQAM) OQAM/FBMC symbols can be formed by modulating each subcarrier with a staggered QAM. The basic idea of FBMC is to transmit real-valued symbols instead of transmitting complex valued ones. Due to this time staggering of the in-phase and quadrature components of the symbols, orthogonality is achieved between adjacent subcarriers.

The synthesized signal burst is therefore a composite of multiple subchannel signals. Each signal consists of a linear combination of time-shifted (by multiples of $T_{s} / 2$ ) and overlapping impulse responses of the prototype filter, weighted by the respective symbol values [2]. Note that each sub-carrier is modulated with an OQAM. OQAM inserts a shift of half the symbol period between the real and the imaginary part of the complex data symbol [2].

In FBMC systems, if the prototype filter with coefficients $b[i]$ with $i=0, \cdots, Y-1$ is used, where $Y=Q N$ and $Q$ is overlapping factor which represents the length of each polyphase components and under the assumption of the even symmetry of prototype coefficients around the $\left(\frac{Q N}{2}\right)^{t h}$ coefficient with zero coefficient in the beginning, the FBMC PSD can be expressed as $\Phi^{n}(f)=\left|B_{n}(f)\right|^{2}$ where $\left|B_{n}(f)\right|$ is the frequency response of the prototype filter and can be written as [2]

$$
\left|B_{n}(f)\right|=b[Y / 2]+2 \sum_{r=1}^{\frac{Y}{2}-1} b[(Y / 2)-r] \cos (2 \pi f r) .
$$

\section{IA BASED CR NETWORK PROBLEM Formulation}

Mostly, frequency division multiple access (FDMA) technique is used to manage the transmission between the SUs in CR network, in which the transmission over each subcarrier is restricted to one SU at a given time. MIMO IA passes over this limitation by giving the opportunity for a number of SUs to share a given subcarrier at the same time. This can be achieved by designing precodeing matrices for the SUs in a way that the desired data is aligned at its own receiver in an interferencefree subspace while the interference signals from other SU transmitters are aligned at the interference subspace [11]. Thus, MIMO IA is proposed to be applied in CR network aiming at improving its throughput under $\mathrm{CR}$ operation constraints. Perfect IA would be proper if $M_{T}+M_{R}-d(K+1) \geq 0$ is satisfied [17]. Therefore, if the number of SUs excesses the feasibility conditions, SUs are clustered into disjoint groups where IA is performed to users within each cluster [18]. In this work, to make the analysis more clear and without loss of generality, a proper CR cluster is assumed in which each SU sends one data stream to its intended receiver (ex. [12]). Accordingly, the discrete-time complex received signal at the $k^{t h}$ receiver over the $n^{t h}$ sub-carrier is represented as

$$
\mathbf{y}_{k}^{n}=\mathbf{u}_{k}^{n \mathrm{H}} \mathbf{H}_{k}^{n} \mathbf{v}_{k}^{n} \mathbf{x}_{k}^{n}+\sum_{j=1, j \neq k}^{K} \mathbf{u}_{k}^{n \mathrm{H}} \mathbf{H}_{k j}^{n} \mathbf{v}_{j}^{n} \mathbf{x}_{j}^{n}+\mathbf{u}_{k}^{n \mathrm{H}} \mathbf{z}_{k}^{n},
$$

where $\mathbf{u}_{k}^{n} \in \mathbb{C}^{M_{R} \times 1}$ is an orthonormal linear interference suppression matrix applied at the $k^{t h} \mathrm{SU}$ receiver, $\mathbf{H}_{k j}^{n} \in$ $\mathbb{C}^{M_{R} \times M_{T}}$ denotes the channel frequency response between $j^{t h} \mathrm{SU}$ transmitter and $k^{t h} \mathrm{SU}$ receiver. All channel state information are assumed to be perfectly known at each node. 


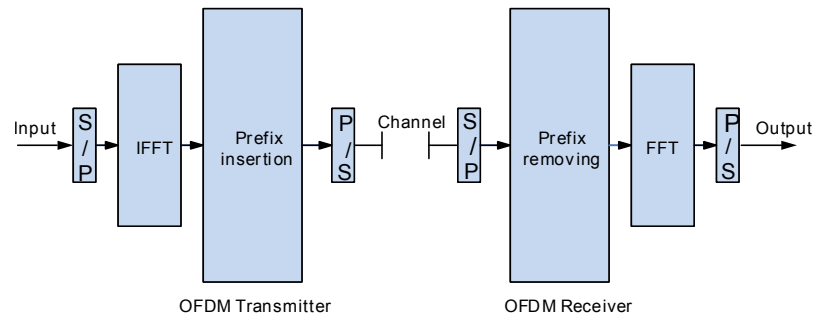

A) OFDM System

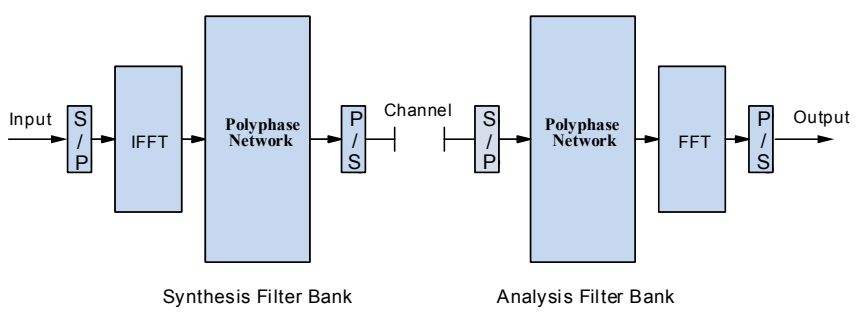

B) FBMC System

Fig. 2: Block diagrams of OFDM and FBMC systems.

$\mathbf{v}_{k}^{n} \in \mathbb{C}^{M_{T} \times 1}$ and $\mathbf{x}_{k}^{n} \in \mathbb{C}$ are the IA precoder matrix and the data stream that is being transmitted from the $k^{t h}$ transmitter, respectively. $\mathbf{z}_{k}^{n} \in \mathbb{C}^{M_{R} \times 1}$ is the zero mean unit variance circularly symmetric additive white Gaussian noise (AWGN) vector at SU receiver $k$.

According to the assumed cluster scenario, IA solution can be obtained with a closed-form solution [11], where the interference is completely eliminated at each SU receiver. Therefore, at the $k^{t h}$ SU receiver, the received signal in (1) becomes

$$
\mathbf{y}_{k}^{n}=\mathbf{u}_{\mathbf{k}}^{\mathbf{n H}} \mathbf{H}_{k}^{n} \mathbf{v}_{k}^{n} \mathbf{x}_{k}^{n}+\mathbf{u}_{k}^{n \mathrm{H}} \mathbf{z}_{k}^{n} .
$$

Moreover, the sum-rate of the SUs over the $n^{t h}$ subcarrier is [19]

$$
R^{n}=\sum_{k=1}^{K} \log \left|1+\frac{P_{k}^{n} \mathbf{u}_{k}^{n \mathrm{H}} \mathbf{H}_{k k}^{n} \mathbf{v}_{k}^{n} \mathbf{v}_{k}^{n \mathrm{H}} \mathbf{H}_{k k}^{n}{ }^{\mathrm{H}} \mathbf{u}_{k}^{n}}{\sigma_{\mathrm{AWGN}}^{2}+\sum_{l=1}^{L} J_{l, k}^{n}}\right|,
$$

where $P_{k}^{n}$ is the transmitted power by the $k^{t h} \mathrm{SU}$ user over the $n^{t h}$ subcarrier and $J_{l, k}^{n}$ is the total interference introduced by the $l^{t h} \mathrm{PU}$ transmitter in the $n^{t h}$ subcarrier to the $k^{t h}$ CR user [20]. $\sum_{l=1}^{L} J_{l, k}^{n}$ can be modeled as AWGN. This is a general assumption in this research area (e.g. [5], [21]). This assumption is justified using the central limit theorem. Therefore, we can write

$$
\sigma_{k}^{n 2}=\sigma_{A W G N}^{2}+\sum_{l=1}^{L} J_{l, k}^{n} .
$$

In CR scenarios, the interference introduced by the $k^{\text {th }} \mathrm{SU}$ transmitter over the $n^{t h}$ CR subcarrier transmission to the $l^{t h}$ $\mathrm{PU}$ receiver should be considered in the problem formulation, which can be expressed as [20]

$$
I_{l, k}^{n}\left(D_{n}, P_{k}^{n}\right)=\operatorname{Tr}\left(P_{k}^{n} \Omega_{l}^{n} \mathbf{G}_{k, l}^{n} \mathbf{V}_{k}^{n} \mathbf{V}_{k}^{n \mathrm{H}} \mathbf{G}_{k, l}^{n \mathrm{H}}\right),
$$

where $D_{n}$ represents the spectral distance between the $n^{\text {th }} \mathrm{CR}$ subcarrier and $l^{\text {th }}$ PU band and $\mathbf{G}_{k, l}^{n} \in \mathbb{C}^{1 \times M_{T}}$ denotes the channel gain between the the $k^{\text {th }} \mathrm{SU}$ transmitter and the $l^{\text {th }}$ PU over the $n^{t h}$ subcarrier. $\Omega_{l}^{n}$ is the interference factor of the $l^{t h}$ subcarrier to the $l^{t h} \mathrm{PU}$, which is obtained by integrating the PSD of the $n^{t h}$ subcarrier over the $l^{t h}$ PU frequency band.

As shown above, MIMO IA is applied on the CR system, where the SUs are able to share all the available subcarriers in the system. Accordingly, SUs are able to share the available spectrum without harming each other. Afterwards, power loading should be peformed for each SU upon the available subcarriers. Power loading is performed in order to maximize the total throughput of the CR system subject to the interference introduced to the PUs and total transmit power budget constraints. Therefore, the problem can be formulated as [15], [16]

$$
\begin{aligned}
P 1: & \max _{P_{k}^{n}} \sum_{n=1}^{N} \sum_{k=1}^{K} \log \left(1+\frac{P_{k}^{n} \mathbf{u}_{k}^{n \mathrm{H}} \mathbf{H}_{k k}^{n} \mathbf{v}_{k}^{n} \mathbf{v}_{k}^{n \mathrm{H}} \mathbf{H}_{k k}^{n} \mathrm{H} \mathbf{u}_{k}^{n}}{\sigma_{k}^{n 2}}\right) \\
\text { s.t. : } & \sum_{n=1}^{N} P_{k}^{n} \leq P_{k} \quad \forall k \\
& P_{k}^{n} \geq 0, \quad \forall n \text { and } \forall k \\
& \sum_{n=1}^{N} \sum_{k=1}^{K} P_{k}^{n} \Omega_{l}^{n} \operatorname{Tr}\left(\mathbf{G}_{k, l}^{n} \mathbf{V}_{k}^{n} \mathbf{V}_{k}^{n \mathrm{H}} \mathbf{G}_{k, l}^{n \mathrm{H}}\right) \leq I_{t h}^{l}, \quad \forall l
\end{aligned}
$$

where (8b) represents the $k^{\text {th }} \mathrm{SU}$ total power constraint $\left(P_{k}\right)$, while a positive transmission power is guaranteed using the constraint in (8c). The constraint in (8d) ensures that the total interference induced by SUs to the $l^{\text {th }} \mathrm{PU}$ is below the prescribed interference threshold $\left(I_{t h}^{l}\right)$.

The problem $P 1$ is a convex optimization problem, which can be solved using the Lagrangian theory. That is the Lagrangian can be written as

$$
\begin{aligned}
G= & -\sum_{n=1}^{N} \sum_{k=1}^{K} \log \left(1+\frac{P_{k}^{n} \mathbf{u}_{k}^{n \mathrm{H}} \mathbf{H}_{k k}^{n} \mathbf{v}_{k}^{n} \mathbf{v}_{k}^{n \mathrm{H}} \mathbf{H}_{k k}^{n}{ }^{\mathrm{H}} \mathbf{u}_{k}^{n}}{\sigma_{k}^{n 2}}\right) \\
& +\sum_{l=1}^{L} \alpha^{l}\left(\sum_{n=1}^{N} \sum_{k=1}^{K} \Omega_{l}^{n} P_{k}^{n} \operatorname{Tr}\left(\mathbf{G}_{k, l}^{n} \mathbf{V}_{k}^{n} \mathbf{V}_{k}^{n \mathrm{H}} \mathbf{G}_{k, l}^{n} \mathrm{H}\right)-I_{t h}^{l}\right) \\
& +\sum_{k=1}^{K} \beta_{k}\left(\sum_{n=1}^{N} P_{k}^{n}-P_{k}\right)-\sum_{n=1}^{N} \sum_{k=1}^{K} P_{k}^{n} \mu_{k}^{n}
\end{aligned}
$$

where $\beta_{k}, \alpha^{l}$ and $\mu_{k}^{n}$ are the non-negative Lagrange multipliers. (9) can be solved as

$$
P_{k}^{n}=\left[\begin{array}{c}
\frac{1}{\sum_{l=1}^{L} \alpha^{l} \Omega_{l}^{n} \operatorname{Tr}\left(\mathbf{G}_{k, l}^{n} \mathbf{V}_{k}^{n} \mathbf{V}_{k}^{n \mathrm{H}} \mathbf{G}_{k, l}^{n}{ }^{\mathrm{H}}\right)+\sum_{k=1}^{K} \beta_{k}} \\
\left.-\frac{\sigma_{k}^{n 2}}{\mathbf{u}_{k}^{n \mathrm{H}} \mathbf{H}_{k k}^{n} \mathbf{v}_{k}^{n} \mathbf{v}_{k}^{n \mathrm{H}} \mathbf{H}_{k k}^{n{ }^{\mathrm{H}}} \mathbf{u}_{k}^{n}}\right]^{+},
\end{array}\right.
$$

where $[y]^{+}=\max (0, y)$. The optimization problem $P 1$ has a high computational complexity which is generally prohibitive 
specially with high number of subcarriers. Therefore, a lowcomplexity sub-optimal algorithm is presented in the next section.

\section{Sub-Optimal Power LoAding Algorithm}

To solve the resource allocation problem $P 1$ efficiently with low computational complexity, a sub-optimal power loading algorithm is proposed.

In our sub-optimal method, we start by finding the solution for the problem $P 1$ after ignoring the per-SU power constraints. Thus, the optimization problem becomes

$$
\begin{aligned}
& P 2: \max _{\widehat{P}_{k}^{n}} \sum_{n=1}^{N} \sum_{k=1}^{K} \log \left(1+\frac{\widehat{P}_{k}^{n} \mathbf{u}_{k}^{n \mathrm{H}} \mathbf{H}_{k k}^{n} \mathbf{v}_{k}^{n} \mathbf{v}_{k}^{n \mathrm{H}} \mathbf{H}_{k k}^{n} \mathbf{u}_{k}^{n}}{\sigma_{k}^{n 2}}\right) \\
& \text { s.t. : } \\
& \sum_{n=1}^{N} \sum_{k=1}^{K} \Omega_{l}^{n} \widehat{P}_{k}^{n} \operatorname{Tr}\left(\mathbf{G}_{k, l}^{n} \mathbf{V}_{k}^{n} \mathbf{V}_{k}^{n \mathrm{H}} \mathbf{G}_{k, l}^{n \mathrm{H}}\right) \leq I_{t h}^{l} \\
& \quad \widehat{P}_{k}^{n} \geq 0, \quad \forall n \text { and } \forall k,
\end{aligned}
$$

where $(\widehat{\cdot})$ indicates the variables that are optimized under the interference constraint only. By solving $P 2$, we get

$$
\begin{aligned}
\widehat{P}_{k}^{n}(l)= & {\left[\frac{1}{\widehat{\alpha}^{l} \Omega_{l}^{n} \operatorname{Tr}\left(\mathbf{G}_{k, l}^{n} \mathbf{V}_{k}^{n} \mathbf{V}_{k}^{n \mathrm{H}} \mathbf{G}_{k, l}^{n \mathrm{H}}\right)}\right.} \\
& \left.-\frac{\sigma_{k}^{n 2}}{\mathbf{u}_{k}^{n \mathrm{H}} \mathbf{H}_{k k}^{n} \mathbf{v}_{k}^{n} \mathbf{v}_{k}^{n \mathrm{H}} \mathbf{H}_{k k}^{n}{ }^{\mathrm{H}} \mathbf{u}_{k}^{n}}\right]^{+}
\end{aligned}
$$

where the Lagrange multiplier $\widehat{\alpha}^{l}$ is evaluated using (12) and (11b) as

$$
\alpha^{l}=\frac{|N K|}{I_{t h}^{l}+\sum_{n=1}^{N} \sum_{k=1}^{K} \frac{\Omega_{l}^{n} \sigma_{k}^{n 2} \operatorname{Tr}\left(\mathbf{G}_{k, l}^{n} \mathbf{V}_{k}^{n} \mathbf{V}_{k}^{n \mathrm{H}} \mathbf{G}_{k, l}^{n}{ }^{\mathrm{H}}\right)}{\mathbf{u}_{k}^{n \mathrm{H}} \mathbf{H}_{k k}^{n} \mathbf{v}_{k}^{n} \mathbf{v}_{k}^{n \mathrm{H}} \mathbf{H}_{k k}^{n}{ }^{\mathrm{H}} \mathbf{u}_{k}^{n}}} .
$$

We assume that the maximum power $P_{k}^{n \max }$ that can be loaded for the $k^{t h}$ SU over the $n^{t h}$ subcarrier can be obtained from the solution of problem $P 2$ as follows

$$
P_{k}^{n \max }=\min \left\{\widehat{P}_{k}^{n}(l)\right\}_{l=1}^{L} .
$$

If the relation $\sum_{n=1}^{N} P_{k}^{n \max } \leq P_{k}$ for all SUs is satisfied, then the solution for $P 1$ is $P_{k}^{n}=P_{k}^{n \text { max }}$. Otherwise, we move to the second step, in which the power budget for each SU $P_{k}$ is distributed among all the subcarriers subject to be lower or equal to the upper-bound of the power of each user at every subcarrier $P_{k}^{n \max }$. The problem can be described as a caplimited waterfilling [22] as

$$
\begin{aligned}
& P 3: \max _{\widetilde{P}_{k}^{n}} \sum_{n=1}^{N} \sum_{k=1}^{K} \log \left(1+\frac{\widetilde{P}_{k}^{n} \mathbf{u}_{k}^{n \mathrm{H}} \mathbf{H}_{k k}^{n} \mathbf{v}_{k}^{n} \mathbf{v}_{k}^{n \mathrm{H}} \mathbf{H}_{k k}^{n} \mathbf{u}_{k}^{n}}{\sigma_{k}^{n 2}}\right) \\
& \text { s.t. : } \quad \sum_{n=1}^{N} \widetilde{P}_{k}^{n} \leq P_{k} \\
& 0 \leq \widetilde{P}_{k}^{n} \leq P_{k}^{n \max },
\end{aligned}
$$

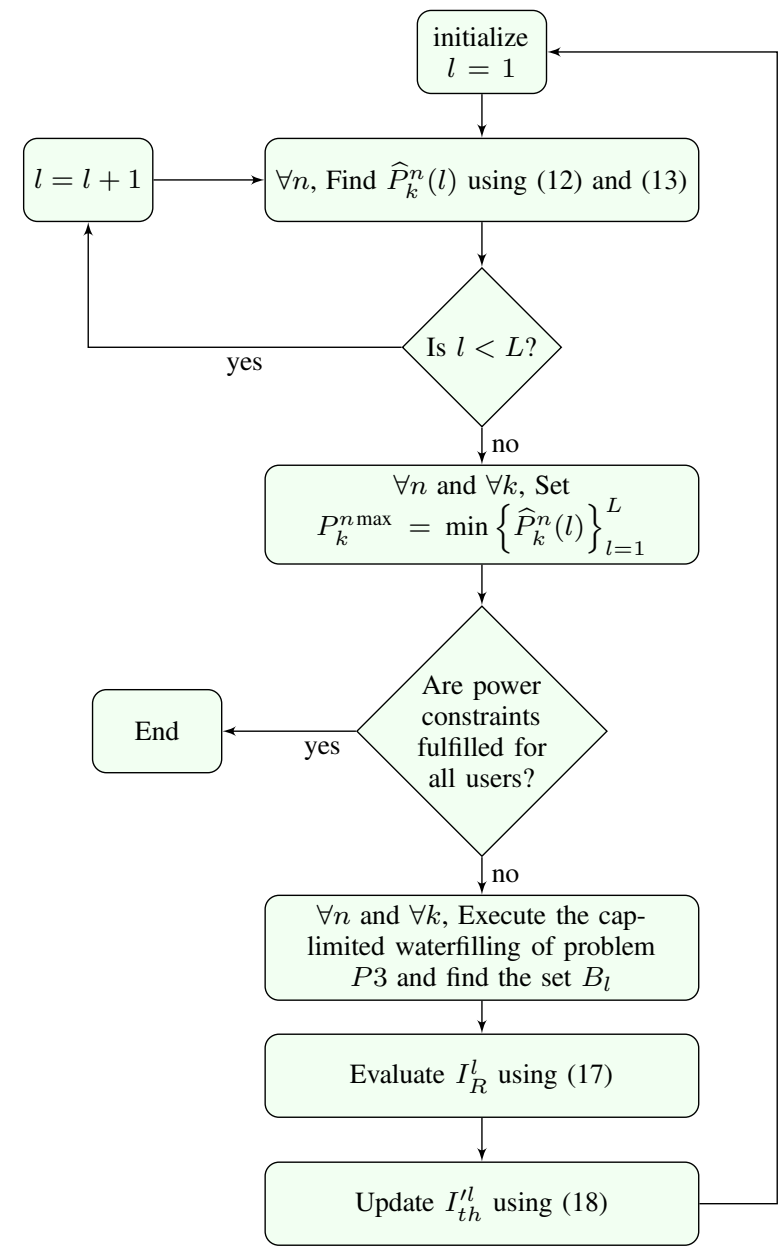

Fig. 3: Flowchart of the sub-optimal power loading algorithm

where $\widetilde{P}_{k}^{n}$ is the solution of problem $P 3$. This problem is solved using the conventional waterfilling, where the waterfilling solution is found as [23]

$$
P_{k, W F}^{n}=\left[\lambda-\frac{\sigma_{k}^{n 2}}{\mathbf{u}_{k}^{n \mathrm{H}} \mathbf{H}_{k k}^{n} \mathbf{v}_{k}^{n} \mathbf{v}_{k}^{n \mathrm{H}} \mathbf{H}_{k k}^{n} \mathrm{H} \mathbf{u}_{k}^{n}}\right]^{+},
$$

where $P_{k, W F}^{n}$ is the allocated power by waterfilling solution for the $k^{t h}$ user at the $n^{t h}$ subcarrier and $\lambda$ is the waterfilling level. For all subcarriers, if the condition $P_{k, W F}^{n} \geq P_{k}^{n \text { max }}$ is satisfied, the power is readjusted to $P_{k}^{n \max }$ and the total power budget is minimized by the difference between them. Then, successive waterfilling is proceeded over the users and subcarriers that have not exceeded $P_{k}^{n \max }$ in the last step until the loaded power $\widetilde{P}_{k}^{n}$ doesn't exceed $P_{k}^{n \text { max }}$ in any user at any subcarrier in the new iteration. In the last step, the allocated power $\widetilde{P}_{k}^{n}$ is lower than the maximum allowed power level as the solution $\widetilde{P}_{k}^{n}$ of $P 3$ problem is constrained to be less than or equal $P_{k}^{n \text { max }}$. This degrades the CR system throughput. To overcome this limitation, the available power gap is re-utilized by allocating some power from one subcarrier to another by re-evaluating the maximum power that can be allocated to each subcarrier $P_{k}^{n \max }$ depending on the residual interference, which can be calculated as

$$
I_{R}^{l}=I_{t h}^{l}-\sum_{n=1}^{N} \sum_{k=1}^{K} \widetilde{P}_{k}^{n} \Omega_{l}^{n} \operatorname{Tr}\left(\mathbf{G}_{k, l}^{n} \mathbf{V}_{k}^{n} \mathbf{S}_{k}^{n} \mathbf{V}_{k}^{n \mathrm{H}} \mathbf{G}_{k, l}^{n} \mathrm{H}\right) \text {. }
$$


Assuming that $B_{l}$ is the set of users that reach the maximum allowed power at some subcarriers, i.e. $\widetilde{P}_{k}^{n}=$ $P_{k}^{n \max }, \forall k$ and $\forall n \in B_{l}$, then, $P_{k}^{n \text { max }}, \forall k$ and $\forall n \in B_{l}$ can be updated by applying the equations (12)-(14) on the users in the set $B_{l}$ with the updated interference constraints, which are

$$
I_{t h}^{\prime l}=I_{R}^{l}+\sum_{n \in B_{l}} \sum_{k \in B_{l}} \widetilde{P}_{k}^{n} \Omega_{l}^{n} \operatorname{Tr}\left(\mathbf{G}_{k, l}^{n} \mathbf{V}_{k}^{n} \mathbf{S}_{k}^{n} \mathbf{V}_{k}^{n \mathrm{H}} \mathbf{G}_{k, l}^{n \mathrm{H}}\right) .
$$

Finally, the procedures of solving problem $P 3$ is re-performed to find the final solution $\bar{P}_{k}^{n}=\widetilde{P}_{k}^{n}$. The flow chart of the proposed suboptimal power loading algorithm is described in Fig. 3.

\section{Simulation Setup and Results}

In our simulation, we evaluate the performance of MIMOOFDM and MIMO-FBMC CR systems using two resource allocation criteria: IA technique and FDMA technique. A feasible IA scenario is simulated in which $K=3$ SUs with $M_{T}=M_{R}=2$ is assumed. The PSD of the $n^{t h}$ subcarrier of the OFDM and the FBMC systems are described in Eq. (1) and Eq. (2) respectively. The values of $N, \Delta f$ and $\sigma_{k}^{n 2}$ are assumed to be 64 subcarriers, $0.3125 \mathrm{MHz}$ and $10^{-6}$, respectively. Two active PU bands are assumed each with $10 \mathrm{MHz}$ bandwidth. Moreover, It is assumed that the channel realizations have been drawn from independent and identically distributed Gaussian distribution with zero mean and unit variance. The results are averaged over 1000 channel realizations.

For the purpose of performance comparison, the following algorithms are considered in the simulation:

1) Optimal IA: find the optimal power loading solution using problem $P 1$ with either OFDM or FBMC physical layer.

2) Optimal FDMA: allocate the power optimally considering a FDMA system as in [1] with either OFDM or FBMC physical layer.

3) Suboptimal IA: perform the power loading based on the algorithm in Fig. 3 with either OFDM or FBMC physical layer.

CVX toolbox is used in our simulation to obtain the optimal solutions [24]. Note that in all the figures, the FBMC simulated results are denoted by dash curves, while the OFDM simulated results are denoted by solid curves.

In terms of complexity, the optimal IA scheme has the complexity of $\mathcal{O}\left(N^{3}\right)$, which is significantly high. Therefore, the sub-optimal approach is proposed with lower complexity. The algorithm in Fig. 3 performs the optimization under only the interference constraint for $L$ primary bands, hence the complexity of this step is $\mathcal{O}(L N \log N) \leq$ $\mathcal{O}(K L N \log N)$. The cap-limited waterfilling is excuted $\mathrm{t}-$ wo times in the algorithm for all SUs with a complexity $\mathcal{O}(K N \log N) \leq \mathcal{O}(K L N \log N)$. The maximum power evaluation depending on the residual interference has a complexity of $\mathcal{O}\left(\left|B_{l}\right| \log \left|B_{l}\right|\right) \leq \mathcal{O}(K L N \log N)$ considering all SUs. Therefore, The complexity of the proposed sub-optimal power loading algorithm is lower than $\mathcal{O}(K L N \log N)$.

Fig. 4 depicts the average sum-rate of the CR system against the interference thresholds. In this figure, the perSU power budget $P_{k}$ is set to be $15 \mathrm{dBm}$. Generally, for

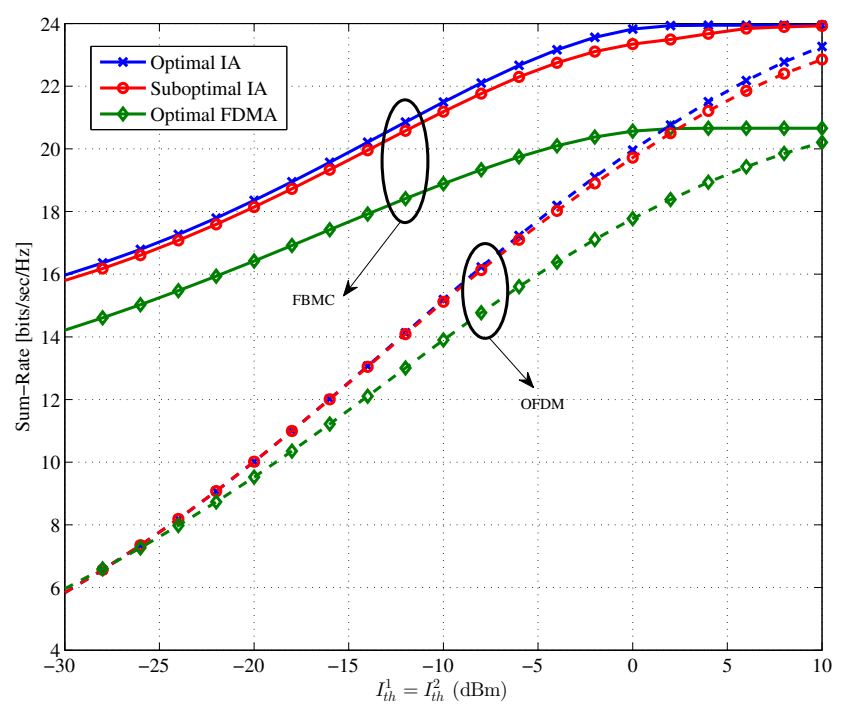

Fig. 4: Sum-rate performance vs. allowed interference limit.

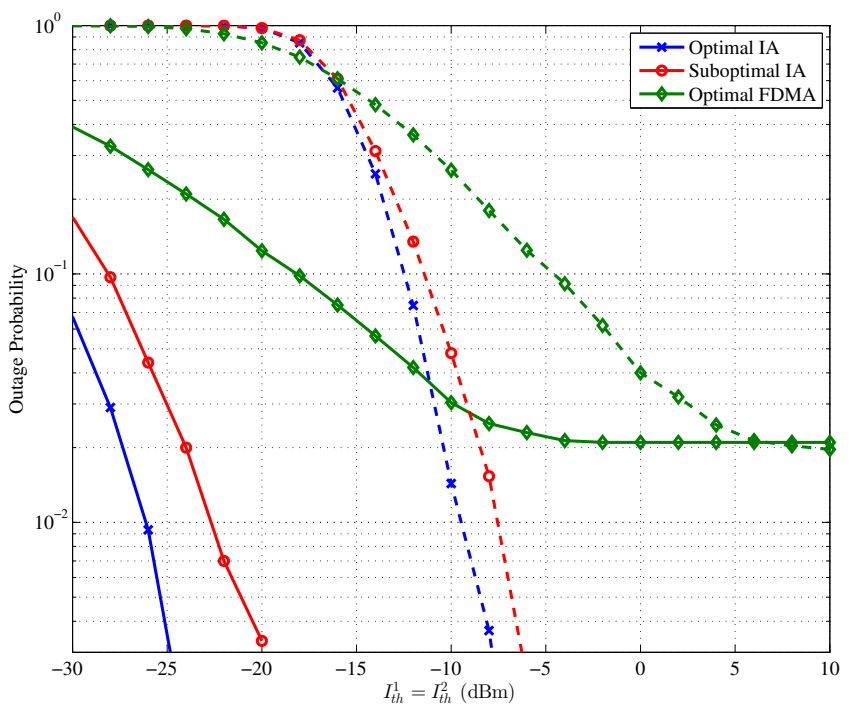

Fig. 5: Outage probability vs. interference limit, $R_{\text {min }}=60 \mathrm{Mbits} / \mathrm{sec}$.

all the simulated scenarios, the average sum-rate increases as the interference threshold levels increase since each SU has more flexibility to allocate more power on its subcarriers. The Suboptimal IA system matches the performance of the optimal $I A$ system in FBMC and OFDM physical layers with fewer complexity. Moreover, it can be observed that the Suboptimal $I A$ algorithm achieves higher sum-rate gain compared to the optimal FDMA algorithm in both physical layer techniques. It is clearly noted from this figure that FBMC multicarrier technique presents better sum-rate performance than OFDM. At very low interference limits, $I_{t h}=-30 \mathrm{dBm}$, Suboptimal IA with FBMC physical layer outperforms Suboptimal IA with OFDM physical layer with approximately $10 \mathrm{bps}$. This gap decreases as the interference limit increases until both systems behave as a non-cognitive system. Moreover, Suboptimal IA gains from FBMC physical layer more sum-rate rather than in OFDM physical layer. As an example, 2 bps is the gap between 
Suboptimal IA and optimal FDMA with FBMC physical layer at $I_{t h}=-30 \mathrm{dBm}$ while both are matched when OFDM physical layer is used. At this end, IA based power loading with FBMC archives a notable sum-rate increase compared to all other simulated techniques. The gain of the FBMC over the OFDM systems returns to the fact that the FBMC system has lower interference to the PU bands than the OFDM system.

Fig. 5 presents the outage sum-rate probability of the different algorithms when the per-SU power budget is fixed to $P_{k}=10 \mathrm{dBm}$, where the minimum rate for each $\mathrm{SU}$ is set to be $60 \mathrm{Mbits} / \mathrm{sec}$, i.e. $R_{\min }=240$ bits per OFDM symbol. Generally, the outage probability decreases as the interference constraint increases since the systems are more able to achieve the minimum instantaneous rate for the different users. It is clearly observed from Fig. 5 that IA approach is able to achieve better sum-rate behavior that traditional FDMA approach. However, the IA based algorithms with FBMC physical layer can mostly achieve the rate target even at very restricted interference limits. Furthermore, the outage probability of the suboptimal is close to optimal IA one in both physical layers, and both are much lower than that of the optimal FDMA.

\section{CONCLUSION}

In this paper, a sub-optimal power loading method based on IA in MIMO-OFDM and MIMO-FBMC CR systems is presented. The problem is formulated so that the total data rate is maximized while the interference introduced to the primary system remains under the prescribed interference limit. Simulations show that the sub-optimal method approaches the optimal sum-rate performance with fewer computational complexity. Furthermore, IA technique achieves a significant sum-rate increase of $\mathrm{CR}$ systems compared to traditional CR systems. Moreover, IA with FBMC physical layer presents the best sum-rate behavior compared to the other simulated scenarios.

\section{ACKNOWLEDGMENT}

This work is funded by German Academic Exchange Service - $(D A A D)$, the French National research Agency (ANR) research project PROFIL with grant agreement code: ANR13-INFR-0007-03, the Spanish Ministry of Economy and Competitiveness (Ministerio de Economiay Competitividad) under project TEC2011-29006-C03-02 (GRE3N-LINKMAC), and from the Catalan Government (2009SGR0891).

\section{REFERENCES}

[1] R. Zhang and Y.-C. Liang, "Exploiting multi-antennas for opportunistic spectrum sharing in cognitive radio networks," Selected Topics in Signal Processing, IEEE Journal of, vol. 2, no. 1, pp. 88-102, 2008.

[2] M. Bellanger, "Specification and design of a prototype filter for filter bank based multicarrier transmission," in Acoustics, Speech, and Signal Processing, 2001. Proceedings. (ICASSP '01). 2001 IEEE International Conference on, vol. 4, 2001, pp. 2417-2420 vol.4.

[3] B. Farhang-Boroujeny, "Ofdm versus filter bank multicarrier," Signal Processing Magazine, IEEE, vol. 28, no. 3, pp. 92-112, May 2011.

[4] R. Zakaria and D. le Ruyet, "A novel FBMC scheme for Spatial Multiplexing with Maximum Likelihood detection," in ISWCS, November 2010.

[5] G. Bansal, J. Hossain, and V. Bhargava, "Optimal and suboptimal power allocation schemes for OFDM-based cognitive radio systems," Wireless Communications, IEEE Transactions on, vol. 7, no. 11, pp. 4710-4718, 2008.
[6] P. Wang, M. Zhao, L. Xiao, S. Zhou, and J. Wang, "Power allocation in OFDM-based cognitive radio systems," in Global Telecommunications Conference, 2007. GLOBECOM '07. IEEE, 2007, pp. 4061-4065.

[7] K. Hamdi, W. Zhang, and K. Letaief, "Opportunistic spectrum sharing in cognitive MIMO wireless networks," Wireless Communications, IEEE Transactions on, vol. 8, no. 8, pp. 4098-4109, 2009.

[8] S. Hua, H. Liu, M. Wu, and S. Panwar, "Exploiting MIMO antennas in cooperative cognitive radio networks," in INFOCOM, 2011 Proceedings IEEE, 2011, pp. 2714-2722.

[9] D. Nguyen and M. Krunz, "Spectrum management and power allocation in MIMO cognitive networks," in INFOCOM, 2012 Proceedings IEEE, 2012, pp. 2023-2031.

[10] G. Scutari and D. Palomar, "MIMO cognitive radio: A game theoretical approach," Signal Processing, IEEE Transactions on, vol. 58, no. 2, pp. 761-780, 2010

[11] V. Cadambe and S. Jafar, "Interference alignment and degrees of freedom of the K-user interference channel," Information Theory, IEEE Transactions on, vol. 54, no. 8, pp. 3425-3441, 2008.

[12] S. Perlaza, N. Fawaz, S. Lasaulce, and M. Debbah, "From spectrum pooling to space pooling: Opportunistic interference alignment in MIMO cognitive networks," Signal Processing, IEEE Transactions on, vol. 58, no. 7, pp. 3728-3741, 2010.

[13] L. Sboui, H. Ghazzai, Z. Rezki, and M.-S. Alouini, "Achievable rate of cognitive radio spectrum sharing MIMO channel with space alignment and interference temperature precoding," in Communications (ICC), 2013 IEEE International Conference on, 2013, pp. 2656-2660.

[14] H. Du and T. Ratnarajah, "Robust joint signal and interference alignment for MIMO cognitive radio network," in Wireless Communications and Networking Conference (WCNC), 2012 IEEE, 2012, pp. 448-452.

[15] M. El-Absi, M. Shaat, F. Bader, and T. Kaiser, "Interference alignment based resource management in MIMO cognitive radio systems," in Proc. IEEE 2014 20th European Wireless Conference (EW2014), May 2014.

[16] M. El-Absi and T. Kaiser, "Optimal resource allocation based on interference alignment for OFDM and FBMC MIMO cognitive radio systems," in Proc. 23rd European Conf. on Networks and Communications (EuCNC'2014), June 2014.

[17] M. Razaviyayn, G. Lyubeznik, and Z.-Q. Luo, "On the degrees of freedom achievable through interference alignment in a MIMO interference channel," Signal Processing, IEEE Transactions on, vol. 60, no. 2, pp. 812-821, 2012.

[18] S. Chen and R. Cheng, "Clustering for interference alignment in multiuser interference network," Vehicular Technology, IEEE Transactions on, vol. PP, no. 99, pp. 1-1, 2013.

[19] K. Gomadam, V. Cadambe, and S. Jafar, "A distributed numerical approach to interference alignment and applications to wireless interference networks," Information Theory, IEEE Transactions on, vol. 57, no. 6, pp. 3309-3322, 2011.

[20] T. Weiss, J. Hillenbrand, A. Krohn, and F. Jondral, "Mutual interference in OFDM-based spectrum pooling systems," in Vehicular Technology Conference, 2004. VTC 2004-Spring. 2004 IEEE 59th, vol. 4, 2004, pp. 1873-1877 Vol.4.

[21] M. Shaat and F. Bader, "Computationally efficient power allocation algorithm in multicarrier-based cognitive radio networks: OFDM and FBMC systems," EURASIP Journal on Advances in Signal Processing, vol. 2010, no. 1, p. 528378, 2010. [Online]. Available: http://asp.eurasipjournals.com/content/2010/1/528378

[22] P. Nikolaos and A. Theodore, "Bit and power allocation in constrained multicarrier systems: The single-user case," EURASIP Journal on Advances in Signal Processing, vol. 2008, 2007.

[23] W. Yu, W. Rhee, S. Boyd, and J. Cioffi, "Iterative water-filling for gaussian vector multiple-access channels," Information Theory, IEEE Transactions on, vol. 50, no. 1, pp. 145-152, 2004.

[24] M. Grant and S. Boyd, "CVX: Matlab software for disciplined convex programming, version 2.0 beta," http://cvxr.com/cvx, Sep. 2013. 